\title{
反应树(Reaction Tree)
}

\section{一归纳总结法在 “药物合成反应”教学中的应用}

叶文静, 丁依婷, 潘洁, 姜军, 赵一玫, 王凯

湖北大学化学化工学院, 武汉 430062

摘要：针对 “药物合成反应” 这一课程有机反应多、化合物结构复杂的特点, 设计用一种 “反应树” 式的归纳总结方法 对教材每个章节的有机反应进行总结。反应树可以清晰地展示同一章节里不同有机反应之间的区别与联系, 使看上去无 规律的有机反应更加系统化、条理化, 从而帮助提高教学效果。

关键词: 反应树; 药物合成反应; 归纳总结法; 大学化学; 教学方法

中图分类号: G64; 06

\section{Reaction Tree: Application of Induction and Summarization in Organic Reactions for Drug Synthesis Teaching}

Wenjing Ye, Yiting Ding, Jie Pan, Jun Jiang, Yimei Zhao, Kai Wang *

College of Chemistry and Chemical Engineering, Hubei University, Wuhan 430062, China.

\begin{abstract}
Organic Reactions for Drug Synthesis" is characterized by many organic reactions and complex compounds, which are difficult for most students to understand. A teaching method of induction and summarization has been described in this article, named "Reaction Tree". A reaction tree can clearly demonstrate the commonalities and differences of different organic reactions. By depicting a reaction tree with important organic reactions in every chapter, the knowledge structures become systematized and methodized, which helps to enhance its effectiveness in teaching these subjects.
\end{abstract}

Key Words: Reaction tree; Organic reactions for drug synthesis; Induction and summarization; University chemistry; Teaching method

“药物合成反应” 是制药工程专业一门重要的专业课, 这门课程是有机化学的加深与拓展, 内 容几乎全是林林总总的有机反应及机理, 相比于有机化学, 药物合成反应涉及到的化合物结构更复 杂, 反应类型更多, 大多数反应还有催化剂加入, 因此更难学。想学好这门课程不能如有机化学一 般单单只记一个反应, 知道反应物和产物就足够, 而是需要掌握规律、寻找共性, 记住一类反应, 因此要求学生能对众多的有机反应进行梳理, 按照一定的规律进行 “归纳总结”, 使零乱、分散的有 机反应形成体系。

\section{1 归纳法的介绍}

正所谓 “工欲善其事, 必先利其器”, 科学的学习方法就像学习过程中的 “利器” 一样, 不但可

收稿: 2021-08-03; 录用: 2021-09-13; 网络发表: 2021-10-25

“通讯作者, Email: kaiwang@ hubu.edu.cn

基金资助: 教育部第二批新工科研究与实践项目(E-HGZY20202020); 湖北大学教学建设项目(202004) 
以提高学习效率又能在有限时间里学习更多的知识。“归纳法” [1]是英国文艺复兴时期哲学家弗兰西 斯·培根提出的科学方法, 是指从现象到本质的分析、具体到抽象的概括、从个别到一般的归纳。利 用归纳法对所学知识进行总结可以化繁为简, 快速了解所学课程的整体框架和精华, 是一种非常高 效的学习方法, 被广泛用在各个领域、学科和课程的学习, 例如语言学、药学、工程、化学等 ${ }^{[2-5]}$ 。

然而, 虽然很多课程尤其是复杂课程的学习中, 很多教师都有提到要对学习内容进行归纳总结, 而且归纳总结确实有效, 但其实大多数学生并不知道如何归纳和总结, 或者说缺少有效的归纳总结 “形式”。这相当于是给学生布置了一个任务一一对知识点进行归纳总结, 但是却并没有告诉学生如 何完成这个任务, 所以学生接到任务时无从下手, 或者只是对知识点进行罗列, 例如抄写书里的有 机反应式, 这种情况只是完成了 “总结” 的过程, 而没有把那些反应总结出规律, 即未进行 “归纳”, 故无法从全局去看所有知识点, 知识仍然是散乱不成系统的。因此, 对于复杂课程, 进行归纳总结 学习很有必要, 但更重要的是要有一种具体的、有效的归纳总结的形式, 这样才能让学生明白教师 下达的任务, 才能发挥出 “归纳总结” 的作用。

\section{2 “反应树(Reaction Tree)” 的介绍及其优点}

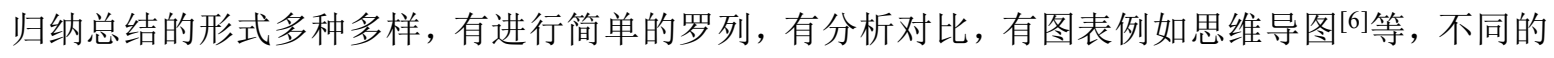
课程适合不同的形式。对课本中的知识点进行简单罗列是最快速、最简单的归纳总结方式, 但这个 过程只是完成了 “归纳”, 却未进行总结, 即缺少 “分析对比” 的过程, 这种方法适用于相对容易学 或章节间逻辑联系不是很紧密的课程。相比较而言, 思维导图是一种更好的归纳总结方法, 将庞大 的信息用图画的方式进行表达, 直观、简洁且生动, 它适用于信息量更大、更难学的课程。而与前 述不同，笔者设想用 “树” 来表达归纳总结的过程，因为树的结构分为树根、树干、树枝和树叶, 是一种很明显的总一分关系, 树根和树干可以看成是 “总”, 而树枝是 “分”, 树叶相当于更精确一级 的 “分”, 一棵树相当于有一个 “树干一树枝-树叶” 这样一个层次分明、层层递进的关系, 实现从个 别到一般的概括, 即 “归纳” 的过程。例如, 英语学习中众所周知的 “Family Tree”, 把家庭成员间 的关系归纳得非常清晰, 很有利于理解记忆。如果将药物合成反应里的有机反应也归纳总结为一棵 树, 我们就可以称之为 “反应树(Reaction Tree)”。

将有机反应用 “反应树” 来归纳总结源于笔者一堂课上的灵感。笔者在备课时就意识到这么多 的有机反应想要记下来而不混淆, 对学生而言是件很困难的事情, 因此一直在思考用怎样的方法帮 助学生记忆。一次在讲绪论时, 为了给学生解释归纳总结的好处, 笔者在黑板上随手画了一棵树, 用树的 “树叶-树枝-树干” 来比喻归纳总结的过程, “我们把每一片树叶看成一个有机反应的话, 那 么树干和树枝就是这些反应的共同之处, 把共同之处与各反应的区别相结合, 就能记住一类反应, 而不是一个或几个反应, 从树叶到树枝、再到树干的不断精炼过程就是归纳总结的过程。” 那一瞬间, 笔者突然意识到, 用一棵树来总结每个章节的有机反应可能真的是一种很好的归纳总结形式, 于是 课后试着画了一下, 发现 “反应树” 可以很好地将章节里看起来散乱的有机反应有条理地进行 “梳 理”, 循着树的 “脉络”, 那些反应之间的区别与联系一目了然, 是一种很有效、也非常适用于药物 合成反应这门课的学习方法。因此在这门课程每个章节学习时, 笔者都会要求学生用一张A4纸画出 这一章的 “反应树”, 大多数学生完成得出乎意料的好, 部分学生反映这种方法确实可以记住更多的 反应, 而且不容易混淆, 甚至有些爱思考的同学画出来的树比我的都清晰、内容更全面, 有的同学 还很有创意, 总结出来的 “反应树” 并不是一个框架, 而是外形像一棵真的树。

\section{3 “反应树” 的画法举例}

目前, 我院制药工程专业选用的教材是闻韧主编的《药物合成反应》第四版 ${ }^{[7]}$, 这本教材有个很 大的优点就是章节是按照反应类型进行划分的, 分为卤化反应、烃化反应、酰化反应、缩合反应、 


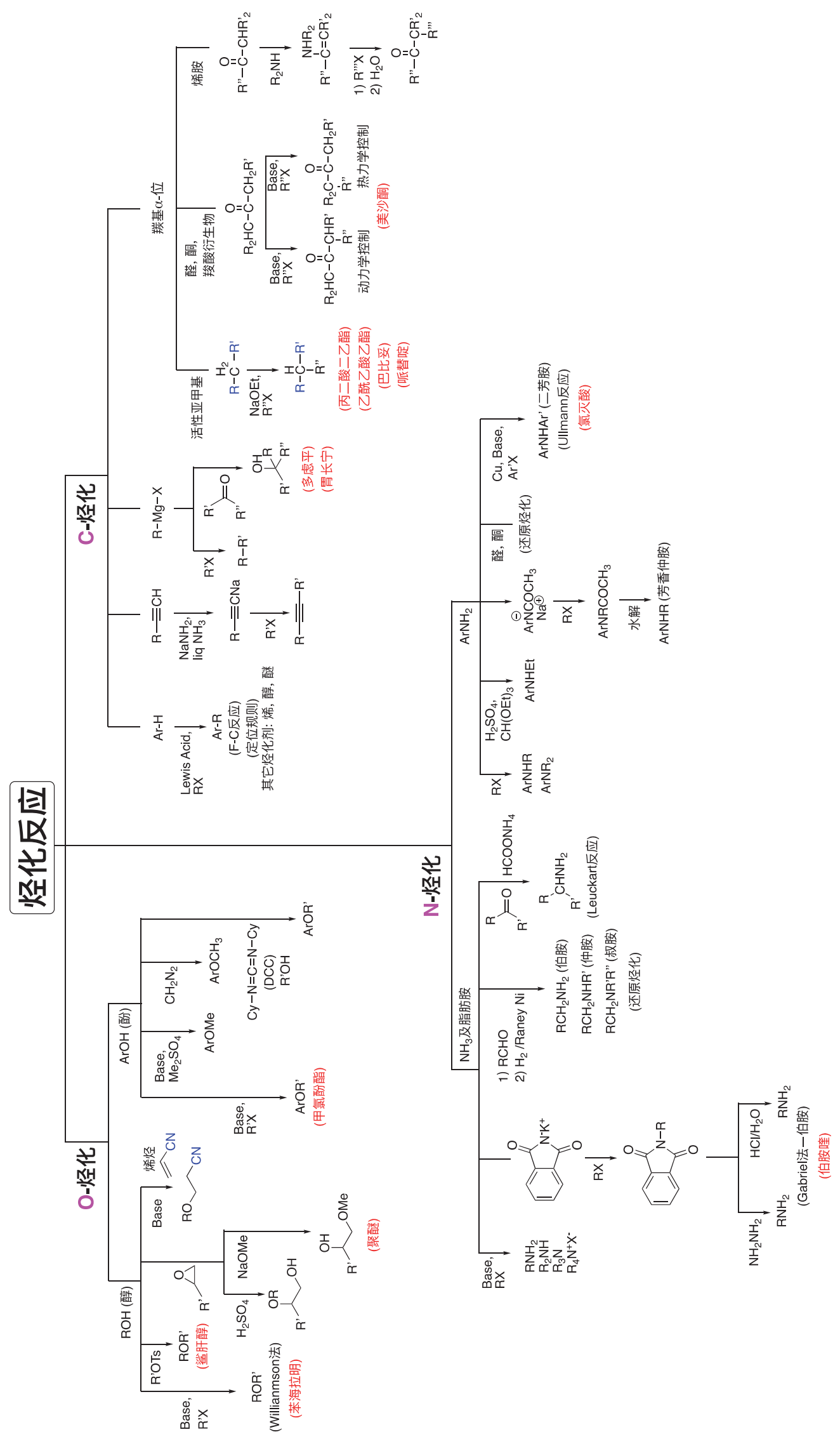

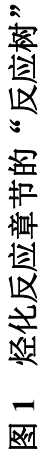


重排反应、氧化反应、还原反应和合成设计原理, 经过这样划分以后, 每个章节的内容之间有着外 在清晰的区别与内在紧密的逻辑联系。首先, 外在区别是试剂结构的区别。药物合成反应的本质是 有机化学反应, 而有机反应主要由 “底物+试剂+催化剂+产物” 组成, 利用 “试剂” 的不同就可以很 清晰地区分不同章节的反应, 例如卤化反应中的卤化剂、烃化反应中的烃化剂和酰化反应中的酰化 剂, 它们在结构上有很大区别, 这样可以避免学生在记忆的时候混淆, 又有利于进行归纳总结。其 次, 内在逻辑联系是它们反应机理上的共通之处。不论是卤化、烃化还是酰化, 涉及的反应机理仍 然主要是亲核和亲电取代或加成, 这又是这几个章节之间的共同点, 所以学生学习了第一章卤化反 应, 再学习烃化、酰化, 就会觉得它们在本质上是相似的, 从而有助于学生理解。例如第一章芳环 卤化、第二章芳环傅-克烃化和第三章芳环傅-克酰化均是芳烃亲电取代反应机理; 第一章崖基 $\alpha$-位卤 化、第二章羰基 $\alpha$-位烃化、第三章羰基 $\alpha$-位酰化以及第四章的许多反应涉及到的都是酸或碱存在下 的烯醇式中间体或碳负离子中间体等等。基于教材的这个特点, 我们在使用 “反应树” 对本书每章 内容进行归纳总结时, 完全是按照书中的章节进行梳理的, 没有也不必重新去分类和归纳共同点。

下面笔者以第二章烃化反应为例, 介绍反应树的画法。如图1所示, 首先, 烃化反应共分为 3 大 节, 从反应树很容易看出, 这3大节的区别是发生烃化的反应原子不同, 分别为 $\mathrm{O}$-烃化、 $\mathrm{N}$-烃化、 $\mathrm{C}$-烃化; 其次, 每一节又分为若干小节, 这是以发生烃化时底物的结构类型决定的, 例如 $\mathrm{O}$-烃化, 含 $\mathrm{O}$ 的底物大家很容易想到醇和酚; 然后, 一类底物的烃化又根据使用不同的烃化剂进行分枝, 比如 醇的烃化有四类烃化剂可以使用, 经过这样一层层梳理下来, 从烃化原子到底物类型再到烃化剂, 相当于对一类底物的烃化反应完成了总结。此外, 反应里需要特别注意的地方还可以用其他颜色字 体标记, 例如对试剂结构有特殊要求的可以用蓝色字体标记, 书中经典的药物合成实例可以用红色 字体列出药物名字, 以方便复习。依此类推, 依据教材的分节很容易就可以画出烃化反应这一章的 反应树。最终, 整个反应树包含反应类型、底物、烃化剂、催化剂和产物, 把课本上几十页的内容 浓缩成一页, 完整地展示了全章内容的框架, 层次分明, 条理清晰, 一目了然。而且, 通过在已有 “树枝” 旁边延伸出新的 “树枝”, 我们可以在这棵反应树里引入更多的细节, 使内容不断丰富, 树 枝不断丰满。

虽然用普通的罗列方法也可对本章内容进行总结, 但并不能很清晰地看出各个有机反应间的区 别与联系, 也更无从去发现规律, 而 “反应树” 不同, 它使这些知识有了层次感, 各反应间的相同 与不同一目了然, 这就像一条 “混乱的队伍” 与一条 “整齐有序的队伍” 的差别, “反应树” 相当于 对 “队伍” 进行了整理, 规律性更强, 更易于被理解、记忆。它与思维导图类似, 也是一种基于图 形的归纳总结方式, 使知识点之间的联系很直观、生动, 但同时它又区别于思维导图, “树干-树 枝-树叶” 这种总分关系更加层次分明, 比思维导图更能体现 “总”与 “分”之间的逻辑联系。

\section{4 结语}

药物合成反应这门课程涉及的内容均是有机反应及机理, 反应类型很多, 进行归纳总结学习非 常有必要, 考虑到课程的特点和教材的编排方式, 笔者认为 “反应树” 式归纳总结具有更突出的优 势, 一方面反应树的绘制可以起到归纳总结法具有的快速熟悉全书内容的作用, 另一方面, 树的 “树 干” “树枝” 就像全书的脉络一样, 对知识点进行了梳理, 使内容层次分明、条理清晰, 这有利于帮 助学生发现各个反应之间的区别与联系以及寻找规律, 从而有助于学生理解、记忆, 这是 “反应树” 式归纳总结法独有的优点。此外, 在描画反应树的时候, 因为需要关注各个化合物的结构、反应位 点, 所以描绘的过程相当于是一次复习, 可以加深学生对化合物的了解, 学生在此基础上再去学习 反应机理, 会感觉更轻松, 而且总结出来的反应树还可以用于日后复习。总体上说, “反应树” 这个 “利器” 能发挥事半功倍的效果。 
[1] 弗兰西斯·培根, 著. 新工具. 许宝骙, 译. 北京: 商务印书馆, 1984.

[2] 丁泽辉. 教育教学论坛, 2020, No. 24, 297.

[3] 杨红艳, 周中流，林三清，夏敬民. 广东化工, 2017, 45 (13), 297.

[4] 张吉玲. 中国电力教育, 2010, No. 32, 84 .

[5] 陈丽琴, 胡志刚. 化学教育, 2013, 34 (6), 47.

[6] 黎卓喜, 董楠, 徐娟娟. 化学教育, 2019, 40 (8), 23.

[7] 闻㓞. 药物合成反应. 第4版. 北京: 化学工业出版社, 2017. 